\title{
Quelques réflexions sur les traductions espagnoles de L'Herbe de Claude Simon
}

\section{Manuel Tost Planet}

\section{(2) OpenEdition}

1 Journals

Édition électronique

URL : http://journals.openedition.org/ccs/945

DOI : $10.4000 /$ ccs.945

ISSN : 2558-782X

Éditeur :

Presses universitaires de Rennes, Association des lecteurs de Claude Simon

\section{Édition imprimée}

Date de publication : 30 avril 2015

Pagination : 61-72

ISBN : 9782753539990

ISSN : $1774-9425$

\section{Référence électronique}

Manuel Tost Planet, "Quelques réflexions sur les traductions espagnoles de L'Herbe de Claude Simon », Cahiers Claude Simon [En ligne], 10 | 2015, mis en ligne le 22 septembre 2017, consulté le 20 avril 2019. URL : http://journals.openedition.org/ccs/945; DOI : 10.4000/ccs.945 


\title{
QUELQUES RÉFLEXIONS SUR LES TRADUCTIONS ESPAGNOLES DE L'HERBE DE CLAUDE SIMON
}

\author{
Manuel TOST PLANET \\ Universitat Autònoma de Barcelona
}

Traduire les romans de Claude Simon constitue une entreprise délicate, compliquée et parfois même risquée. Tous les traducteurs (professionnels ou occasionnels) que j'ai consultés sur la question ont corroboré cette affirmation, à quelques nuances près: pour certains il est question de trouver les clefs d'une variété commune de textes appartenant à un moment précis de la littérature française du xx $x^{\mathrm{e}}$ siècle (ce qu'on a pu appeler le Nouveau Roman); pour d'autres, d'une manière plus précise, il s'agit surtout de s'habituer au rythme de la prose simonienne, à sa musicalité.

Quand je parle des traducteurs professionnels de Claude Simon que je connais (ou que j'ai connus), je me réfère en particulier à Manuel Serrat Crespo (Le Palace), avec qui je collabore dans un jury de traduction; à José (Pepe) Escué, récemment décédé, qui était agrégé de l'enseignement secondaire espagnol (et a traduit Histoire, L'Acacia, Les Géorgiques); ou bien encore à Javier Albiñana (Le Tramway), lui aussi enseignant du secondaire, que je ne connais qu'indirectement.

Je dois dire encore que si j'avais étudié un autre ouvrage de Claude Simon (Le Palace, Les Géorgiques ou Le Tramway), il m’aurait été possible de me référer également aux traductions en catalan, mais il se trouve que, à ma connaissance du moins, L'Herbe n'a pas été traduit dans cette langue, pas même Le Vent (dont la traduction espagnole publiée à Buenos Aires est aujourd'hui introuvable), alors que ces romans sont précisément situés dans des régions 
proches de la frontière espagnole, en Catalogne Nord, en quelque sorte du moins pour Le Vent.

Il faut encore ajouter que Robin Lefère, de l'Université Libre de Bruxelles, a fait paraître un article dans une publication de l'université de Murcie, précisément sur le thème de "La traducción al español de las novelas de Claude Simon ». Lefère y présente un état des lieux, à ce moment-là, des traductions en espagnol (il y a vingt ans), il tente une énumération des principales difficultés auxquelles les traducteurs ont été confrontés et s'attache, en passant, à définir les traits de la phrase simonienne:

I) Les textes grammaticalement les plus caractéristiques présentent une phrase très longue, avec une syntaxe très flexible mais surtout très compliquée et embrouillée à cause des nombreuses incises (parenthèses et tirets) et incises dans les incises (voir par exemple Le Vent, L'Herbe, Les Géorgiques...). Il en résulte que le lecteur peut se perdre, se perd, et le traducteur lui-même peut se tromper (p. 87) ${ }^{1}$.

Un peu plus loin, Lefère fait une allusion concrète au texte qui nous occupe. Et je dois dire que je partage nombre des affirmations de l'ensemble de l'article, encore que je pense que l'on peut raisonnablement aller plus loin dans certaines des analyses.

Cela étant, je crois en premier lieu que l'on doit prendre en compte obligatoirement le fait que L'Herbe se situe à un point crucial de l'évolution du style narratif de Claude Simon. Souvenons-nous: "Avec L'Herbe », dit Simon, « il me semble que quelque chose d'assez différent s'est produit. Un tournant était pris ${ }^{2}$.» À partir de ce moment (ou peut-être même, selon nous, avec Le Vent) ${ }^{3}$, Claude Simon a abandonné un style plutôt conventionnel pour aborder une écriture littérairement singulière. C'est là, à n'en pas douter, un aspect important de ces romans.

Après avoir traité brièvement des traducteurs (l'approche externe de la question, en quelque sorte) nous aborderons quelques questions, plutôt internes dans

1. Texte original: "Los textos gramaticalmente más característicos presentan una frase larguísima, con una sintaxis muy flexible pero sobre todo enredosa y enmarañada por los numerosos incisos (paréntesis o rayas), e incisos dentro de incisos (véanse por ejemplo Le Vent, L'Herbe, Les Géorgiques...). Resulta que el lector se puede perder, se pierde, y que el mismo traductor se puede confundir ». Anales de Filología francesa, no 6, 1994 (p. 85-93),

2. «Réponses de Claude Simon à quelques questions écrites de Ludovic Janvier ", Entretiens, 31, 1972, Rodez, Éditions Subervie, p. 17.

3. C'est, en tout cas ce qu'avait bien vu l'auteur de la célèbre Grammaire du français classique et moderne: " [L'emploi presqu'exclusif] du participe présent par $\mathrm{Cl}$. Simon donne la clé du ton plein de rumeurs, où naît, ondule et se dénoue l'admirable récit intitulé Le Vent. » R.-L. Wagner, La Grammaire française. Les niveaux et les domaines. Les normes. Les états de langue. (Paris, SEDES, 1968), p. 68. 
ce cas à la problématique: a) la perspective narrative (signes de ponctuation et pronoms de la première personne) et questions traductologiques touchant au lexique; b) des questions de rythme et de sonorités, que l'on pourrait sous-titrer " Participer et être présent »; et c) le micro-système de la négation dans le début du roman.

\section{LES TRADUCTIONS DE L'HERBE EN ESPAGNOL}

Il y a donc deux versions de L'Herbe en espagnol. Celle de Miguel Angel Asturias et Blanca de Asturias (qui date de 1961, c'est-à-dire de trois ans après sa publication en français), par Fabril Editora de Buenos Aires.

Peut-être un peu oublié maintenant, Miguel Angel Asturias (1899-1974) a été un auteur célèbre en son temps. Poète, romancier, créateur d'une prose lyrique en prise sur les légendes de sa terre d'origine, ce Guatémaltèque, en plus de sa fonction de diplomate, sera lui aussi prix Nobel de Littérature, mais bien avant Claude Simon, en 1967. Dès lors, comment interpréter le fait que M. A. Asturias se soit chargé de traduire L'Herbe? On ne sait. Est-ce par intérêt pour ce roman ou bien pour d'autres raisons? Il faut se souvenir en tout cas que c'est durant son exil en Argentine, de 1954 à 1965 et avant qu'il ne soit nommé ambassadeur de son pays en France (où il avait d'ailleurs fait un séjour d'études auparavant et où il avait rencontré André Breton) que M. A. Asturias et sa femme traduisent le roman de Claude Simon. Peut-être est-ce tout simplement à cause d'une situation économique difficile à ce moment-là? On ne sait. Un an avant le Nobel, en 1966, il avait reçu le prix Lénine de la paix. Il est mort à Madrid en 1974.

D'autre part, il y a celle d'Esteban Busquets et Esther Tusquets (1986, vingtcinq ans plus tard), chez Lumen. Esther Tusquets est décédée l'année dernière, à Barcelone, à l'âge de soixante-seize ans. Elle était directrice des Éditions Lumen (qui ont publié un certain nombre d'ouvrages de Claude Simon); elle était ellemême écrivaine et a publié plusieurs romans, dont l'un a reçu le Prix de la Ville de Barcelone.

Il convient, je crois, de dire quelques mots sur ces deux couples de traducteurs. Blanca Mora y Araujo de Asturias, de nationalité argentine, était la deuxième épouse de M. A. Asturias. Ensemble, ils ont signé au moins deux autres traductions: Le Labyrinthe, de Robbe-Grillet (en 1962), après avoir traduit Les Sequestrés d'Altona, de J.-P. Sartre (en 1961).

Pour tout dire d'entrée, leur traduction de L'Herbe laisse beaucoup à désirer et l'on se demande même dans quelle mesure on peut attribuer la paternité réelle 
de la version du roman simonien à $\mathrm{M}$. A. Asturias. Car, que l'on sache, une traduction directe (version) est, par définition, un exercice de langue maternelle et si l'on se souvient qu'il a été lui-même plus tard prix Nobel de littérature et, donc censé être un expert en langue espagnole avec une bonne connaissance du français (compte tenu de ses séjours en France), on finit par se poser des questions sur cette traduction déficiente.

Pour l'autre couple, on peut aussi parler de symétrie inversée, l'écrivaine reconnue et directrice des éditions Lumen étant Esther Tusquets. Pour la traduction de ce couple, ce que l'on peut dire est que leur texte semble d'abord très redevable de celui de Blanca Mora d'Asturias, amélioré certes, mais encore assez fautif. Il semble, d'autre part, que la traduction serait plutôt le fait d'Esteban Busquets (croyons-nous), dont la compagne dit quelque part qu'il est le père de ses enfants, mais l'est-il également de la traduction? On ne sait. Busquets est surtout connu pour une Anthologie de Mafalda de Quino.

Ainsi donc, ces deux traductions par des traducteurs différents (c'est le seul cas dans les traductions des romans de Claude Simon en espagnol) sont à notre avis plutôt mauvaises, l'une étant inspirée de l'autre, et qui pourraient bien être (pour les deux) l'œuvre du deuxième signataire des deux couples, avec la caution du premier, écrivain beaucoup plus connu, dans chaque cas. Encore faut-il que nous fondions, par des exemples concrets, cette affirmation quelque peu péremptoire.

\section{PERSPECTIVE NARRATIVE ET PONCTUATION}

Le roman commence, comme on sait, in medias res et les personnages ne sont pas nommément désignés d'emblée, mise à part Louise; on ne découvre (dans le double sens du mot) leur identité que progressivement. Claude Simon est d'ailleurs un habitué du fait, les "elles », les "ils ", sont nombreux dans ses incipits. Songeons au "l'une d'elles...» d'Histoire qui phonétiquement peut se lire de différentes manières /lune d'ailes/ ; ou bien encore aux trois "ils " du début des Géorgiques dont les référents renvoient à des personnages historiques très différents. Or les langues romanes autres que le français se distinguent de ce dernier par la non obligation de l'énonciation des pronoms sujets, ce qui fait que quand les pronoms personnels sujets sont énoncés, ou bien cela semble peu naturel, ou bien c'est que l'on tient à en faire une marque d'insistance. Ce qui, bien évidemment, va à l'encontre de l'effet recherché par Claude Simon.

Dans les premières lignes du roman original, il n'y a pas moins de douze (12) « elle »; dans la traduction, il en reste sept (7), « ella », alors qu'on pourrait nor- 
malement en supprimer cinq (5), dont l'antécédent est Marie, et deux (2) dont l'antécédent est Louise (ceux-là, il faudrait sans doute les conserver).

L'abondance de ces pronoms a un effet (recherché, à mon avis) chez Claude Simon de mise en attente (et à distance), quelque part de suspense narratif, ce qui permet de situer sur le même plan Marie et Louise. Ce qui est à l'opposé de ce qui se passe en espagnol où la réitération devient une forte marque d'insistance.

Tusquets, sur ce point, est un peu plus vigilante qu'Asturiascar elle corrige l'usage erroné de la forme du passé, qui dénote (ou suggère) la mort préalable d'une Marie encore agonisante:

\section{As. habria llorado >}

As. habría permitido llorarse $>$

As. Pero ella no era nada tuyo >

\section{TU. Lloraría}

Tu. permitiría llorarse;

Tu. Pero ella no es nada tuyo.

Une chose aussi anodine ou aussi simpleque la ponctuation donne également des indications précises sur le mode de fonctionnement du discours romanesque.

Claude Simon ouvre les guillemets pour indiquer le discours direct d'une personne, en l'occurrence de Louise. Puis, avec le tiret, on passe à l'amant, l'interlocuteur de Louise. Mais quand les guillemets se ferment, après - Non ", répéta-telle..., c'est une autre voix narrative qui prend le relais, l'énonciateur, cette " voix anonyme [qui] parle à la troisième personne ", qu'évoque Alastair B. Duncan dans la Notice de la "Pléiade 4 » et que Gérard Roubichou ${ }^{5}$ avait appelé le "narrateur étranger à la fiction " (mais le mot narrateur ne plaisait pas à Claude Simon). Puis, de nouveau, les guillemets ouverts, "Alors. - Alors rien ", dit-elle... Nouvelle notation entre parenthèses, qui peut être mise de nouveau sur le compte de la voix off de l'énonciateur (de la "voix anonyme "), avant que Louise ne reprenne: "Rien: elle ne s'est jamais... ", etc. Ne nous trompons pas, tout cela n'est pas indifférent.

En l'espace de quelques lignes, nous avons pratiquement l'ensemble des personnages du roman (hormis Sabine) et les différents plans du discours narratif et cela est parfaitement indiqué dans la version originale. Si on avait à lire le texte à haute voix, il faudrait sans doute réserver aux interventions de la voix anonyme un autre ton que ceux de Louise et de son amant. Ce sont ces différents plans narratifs qui, à notre avis, donnent sa tonalité particulière à L'Herbe, et constituent le « tournant " pris... dont il a été question plus haut.

4. Claude Simon, Euvres, t. II. "Bibliothèque de la Pléiade ", n 586, Alastair B. Duncan: Notice de L'Herbe, p. 1385.

5. G. Roubichou, Lecture de L'Herbe de Claude Simon. Lausanne, L'Âge d'Homme, 1976, p. 68. 
Cependant, cela n'est pas rendu dans les traductions: ni dans celle de Tusquets et encore moins dans celle d'Asturias où tout est mis sur le même plan et où la ponctuation est mal transcrite.

\section{LES SONS EN $[\tilde{a}]^{6}$}

En second lieu, je voudrais ajouter quelques mots - après bien d'autres d'ailleurs $^{7}$ - sur l'usage du participe présent chez Claude Simon, cette fois dans L'Herbe (et en rapport avec ses traductions espagnoles), parce qu'il me semble que c'est surtout dans ce roman que l'auteur systématise un usage de cette forme verbale qu'il avait commencé à pratiquer dans Le Vent. Je le ferai pour insister sur l'affirmation faite plus haut que la prose de ce roman devrait être lue à haute voix.

Pour les traductions, était-il possible d'utiliser, dans tous les cas, l'équivalent (approximatif) castillan du participe présent français? Oui, à ceci près que son usage aurait été beaucoup moins naturel. En espagnol, le participe présent, en tant que tel, n'existe pas, c'est le gerundio (le gérondif) qui est utilisé le plus souvent, mais avec des différences d'emploi et surtout de fréquence ${ }^{8}$. Le résultat est que le rythme particulier de la prose simonienne disparaît; ce discours ininterrompu, sans solution de continuité, marqué par une sorte de conflit entre le sémantisme des verbes et la valeur aspectuelle (permanente) du participe cesse de fonctionner, s'arrêtant, s'interrompant... D'autant que si les traducteurs avaient maintenu le gérondif espagnol, il aurait fallu qu'ils postposent pour les pronominaux le pronom réfléchi correspondant, deteniéndose, interrumpiéndose, quedándose, ce qui aurait donné une autre sonorité.

6. Y valga la redundancia, dirait-on en espagnol pour signifier le redoublement.

7. Citons au moins, B.T. Fitch, "Participe présent et procédés narratifs chez Claude Simon », Revue des Lettres modernes, no 94-99, 1964; D. Zemmour, «Le participe présent dans La Route des Flandres. Écriture du souvenir et quête de l'instant ", L'information grammaticale, Vol. 76, no 76, 1998, p. 42-45; H. Weinrich dans sa Grammaire textuelle du français, 1989, Didier/Hatier, écrit: "En employant un participe neutre, [participe présent] le locuteur marque l'écart de son style par rapport à la langue de tous les jours. Ceci peut être l'expression d'un style particulièrement soigné... » (p. 309).

8. Pour P. Gerboin et C. Leroy (Grammaire d'usage de l'espagnol contemporain, Hachette Éducation, 1991) les traductions de cette forme verbale en espagnol répondent aux normes suivantes: "a) le participe présent français qui a une valeur de proposition relative - il exprime une qualité et non pas une action - se traduit en espagnol par une proposition relative [...]; b) le gérondif espagnol n'est pas apte en principe (souligné par nous) à traduire le participe présent français [...]; le participe présent français, employé dans une proposition en apposition, en tête de phrase, se rapporte au sujet de la phrase: il se traduit donc en espagnol par le gérondif $[. .$.$] ; d) le participe présent français employé avec une valeur absolue est traduit en espagnol par une$ proposition subordonnée circonstancielle" (pages 243-244). Comme on peut le constater, cette traduction est plutôt délicate. 
Le participe présent produit également un effet de rythme, comme l'indique justement l'auteur du mémoire sur " l'usage du participe présent dans L'Invita$t_{\text {tion }}{ }^{9}$ ". La présence du participe présent est redoublée, renforcée, dans sa désinence, par l'abondance (qui ne peut pas être fortuite) de vocables comportant le phonème nasal [ã] : maintenant, brusquement, tant, rageusement, dans, impuissant, etc., le plus souvent (pas toujours) en fin de groupe rythmique, qui relaient et confortent la tonalité du passage.

Sans entrer dans des développements phonostylistiques, il faut en conclure qu'il y a de grandes difficultés à rendre cela en espagnol, car comme l'on sait, la modalisation nasale des voyelles est un des traits les plus caractéristiques des réalisations phoniques du français. Nous voulons dire une fois encore par-là que la prose de Claude Simon est, selon nous, à lire à haute voix et, pour distinguer les divers plans de l'énonciation (que ce soit le monologue plus ou moins intérieur de Louise ou le discours de l'énonciateur), il y aurait lieu de varier les locuteurs.

Rendre perceptibles les impressions ou réactions de Louise, sa véhémence, la crise qu'elle traverse, en ce début de roman, les états d'âme qui l'empêchent de partir, de quitter Georges (cela nous le saurons plus tard dans le texte), se fait non seulement à l'aide du sémantisme des mots, mais encore par les sonorités du discours, par le rythme de la prose. Quelque part, Claude Simon a dit que, dans La Route des Flandres, il avait souhaité faire entendre le son du canon (comme Conrad avait fait entendre la voix du nègre dans Le Nègre $d u$ Narcisse... $)^{10}$. Et Conrad ajoutait:

Tout art doit s'adresser d'abord aux sens, et une conception artistique qui s'exprime à l'aide de mots écrits doit s'adresser aux sens, si son intention profonde est d'atteindre la source même de nos émotions. Il lui faut aspirer de toutes ses forces à la plasticité de la sculpture, à la couleur de la peinture, à la suggestion magique de la musique, cet art des arts ${ }^{11}$.

Il y a beaucoup d'autres difficultés de traduction présentes dans les premières pages de L'Herbe qui mériteraient qu'on s'y arrête:

9. L. Rönnholm, L’usage du participe présent dans L'Invitation de Claude Simon et sa traduction suédoise. Youlukuu, 2009.

10. «Dans La Route des Flandres, il y a quelque chose que je ne suis pas arrivé à rendre comme je voulais. J'ai d'abord cru que c'était la peur et l'angoisse, mais c'était idiot de dire ça, j’ai longtemps cherché et j'ai trouvé: c'était le son du canon! Le son exact du canon! Dans Le Nègre du Narcisse, au début, Conrad décrit la voix du nègre; eh bien! lorsqu'on lit ça, on " entend » la voix du nègre... C'est sensationnel! C'est cela que j'aurais voulu: faire entendre le bruit du canon, cette espèce de boum-boum d'une porte qui claque dans une maison vide. Le son du canon, je l'ai raté, j’y arriverai! » (Madeleine Chapsal: « Entretien. Claude Simon parle ». L'Express, 5 avril 1962.

11. J. Conrad, préface du roman Le Nègre du "Narcisse », édit. de La Nouvelle Revue Française, Paris 1924. 
Des ajouts:

dans sa paume ouverte As. en la palma abierta de su mano (p. 15)

n'achevant pas as. no terminaba la frase (p. 15)

Des variantes:

cessant même de frapper as. dejando también de golpearse (p. 15)

Des ruptures de construction. Dans l'une du moins des traductions plus que dans l'autre: la version de l'incise du début du texte:

\section{ORIGINAL}

(à force de réfléchir au meilleur moyen de porter une robe à peu près trois fois plus de temps qu'il n'en faut pour s'user jusqu'à la trame au tissu dont elle a été primitivement faite)
AS.

(a fuerza de pensar en el mejor modo de llevar un traje tres veces más tiempo del que resistía, hasta gastar la trama misma de la tela en que primitivamente había sido hecho)

TU.

(a fuerza de pensar en el mejor modo de llevar un traje tres veces más tiempo del que se necesitaba para gastar la trama misma de la tela en que primitivamente había sido hecho)

Ce n'est certes pas très important, il n'empêche que l'expression descriptive (s’user jusqu’à la trame) ne semble pas avoir été comprise, soit que les traducteurs l'aient ignorée soit qu'ils n'aient pas saisi la construction quelque peu complexe de la phrase (à peu près trois fois plus de temps qu'il n'en faut [au tissu dont elle a été primitivement faite] pour s'user jusqu'à la trame.)

À propos de la désignation des personnages, nous avons également noté un aspect qui soulève habituellement des polémiques en traduction: l'adaptation ou non (pour la couleur locale) des prénoms ou des noms propres (Louise / As. Luisa / TU. Louise), plus loin (Georges / As. Jorge / TU. Georges). Les deux traducteurs donnent des versions différentes, on ne sait justifiées sur quoi.

\section{LE MICROSYSTÈME DE LA NÉGATION EN FRANÇAIS ET LA THÉMATIQUE DOMINANTE DE L'HERBE}

Pour terminer, on me permettra de revenir à l'incipit et de dire quelques mots sur la présence particulièrement prégnante des formes négatives dans le début du 
roman. Le microsystème presque entier de la négation en français est présent: négation totale, négation partielle, intégrée ou non, négation de l'humain, de l'inanimé, temporelle... Et le français se caractérise, comme on sait, par la double particule négative, ce qui détermine un effet d'insistance remarquable.

Il y a aussi un contre-sens manifeste d'Asturias, dans la première ligne de sa traduction (ninguno, en espagnol, négation de la classe, impliquant un pluriel antérieur, rétroversion: aucun). Cette forme est très mal venue si l'on considère que, précisément, Marie n'a personne (forme négative de l'humain qui étonne toujours les apprenants de la langue française).

Là aussi, les traductions (Tusquets ne commet pas la même erreur qu'Asturias) perdent certains des effets produits par les caractéristiques de la langue d'origine et recherchés, me semble-t-il, par Claude Simon. Cette pléthore négative accompagne l'idée (le thème) de la mort, omniprésente en cette ouverture du roman, et le fermera, comme nous l'avons dit, par un procédé très proustien: la fin renvoyant à son recommencement et ainsi de suite... La formule négative qui commence le roman, Mais elle n'a rien, le bouclera: puis plus rien. (Dans Le Palace, c'étaient les odeurs qui suggéraient la mort.)

\section{CONCLUSION PARTIELLE}

Le principal intérêt de notre exercice d'analyse des traductions espagnoles du début de L'Herbe, de Claude Simon, résidait - selon nous - dans le fait de comparer deux versions différentes dont les auteurs étaient plus connus comme écrivains que comme traducteurs; que ces deux versions avaient certes entre elles une distance d'un quart de siècle, mais cette donnée ne pouvait déterminer des différences notables quant aux états de langue respectifs (ce qui est souvent un phénomène déterminant dans les variations constatées des traductions d'ouvrages des temps passés). En revanche, les deux versions avaient un net facteur différentiel dans leur origine: espagnol d'Amérique et espagnol de la péninsule ibérique. Cela admis, la proximité des langues, français-espagnol, n'aurait pas dû poser de graves difficultés. Et pourtant...

On voit bien sur quel type de conclusion (partielle) on va déboucher ici : pour prendre tout l'intérêt, tout le plaisir à la lecture des textes de Claude Simon, il faut bien sûr les lire dans le texte; les traductions dénaturent ou détruisent souvent une part de sa richesse. C'est d'ailleurs peut-être ce qu'ont pensé les éditeurs de catalan (à moins que ce ne soit pour d'autres raisons, moins avouables, de type économique). 
Heureusement, les traductions d'autres romans de Claude Simon sont nettement meilleures, sur tous les plans, que celles de L'Herbe. On pense en particulier au Palace, à Histoire ou aux Géorgiques. En dépit des particularités du discours simonien, les traductions sont plus proches de l'original, tant en espagnol qu'en catalan.

Sans doute une meilleure connaissance de l'œuvre de Claude Simon a-t-elle été déterminante pour que - au fur et à mesure que l'on avançait dans le temps les traductions des romans simoniens aient été améliorées et plus soignées. Cela est vérifiable de la première à la deuxième version de L'Herbe, comme on a pu le voir, mais cela l'est également dans les traductions en espagnol les plus récentes.

En catalan, c'est différent, et c'est dans cette langue que l'on souhaiterait maintenant pouvoir lire des traductions nouvelles, alors que notre auteur a eu depuis toujours de puissants liens avec la Catalogne Sud et qu'on a même pu dire de lui (certainement de manière un peu forcée) qu'il était "l'unique prix Nobel catalan ».

\section{APPENDICE: QUELQUES EXEMPLES DE TRADUCTION (LEXIQUE)}

Original

\section{Divergences}

a fouillé

marchands de bestiaux

biscuits

berlingots

en fer

piquetée

à demi allongée

volant

$\varnothing$

couché
As.

Tu. registró

troperos (americanismo)

bizcochos

confites

latón

agujereada

acostada

volado (americ.)

pollera (americ.)

acostado revolvió

ganadero

galletas

caramelos

metal (hyperonyme)

punteada

recostada

volante

falda

tendido 


$\begin{array}{lll}\text { cadre } & \text { encuadre (americ. }) & \text { marco } \\ \text { nœuds } & \text { mońos (americ. }) & \text { lazos }\end{array}$

\section{Réalités différentes}

coffret à bijoux

boîte à biscuits

vêtue d'une longue

robe blanche

à demi allongée cofre de joyas

arquilla, estuche de joyas, cofre

joyero

vestida con un largo

vestido blanco

acostada

(retroversión: couchée) (retroversión: coffre et non coffret)

vestida de un largo

traje blanco

recostada

(retroversión:

appuyée, adossée)

\section{Variations}

petits chiens blancs frisés perritos blancos y lanudos

caniche

bleu pervenche

prairie perrito (caniche, la race ne perrito

figure que depuis peu dans le (voir ci-contre) dictionnaire)

color celeste

prado perritos blancos $\mathrm{y}$

lanudos

color celeste

pradera

\section{Autres ruptures de construction}

pudiques et ridicules

(l'original renvoie aux (la traduction renvoie à la souliers dépassant le vo- pollera, fem. sing.) lant de la robe) púdica y ridícula

púdica y ridícula

(la traduction renvoie à la falda, ferm. sing.) 


\section{SUR LE PARTICIPE PRÉSENT}

Pour conforter l'affirmation faite plus haut sur le fait que la prose de ce roman devrait être lue à haute voix. Voici quelques données touchant à l'utilisation des formes verbales au début du roman:

\begin{tabular}{|c|c|c|c|}
\hline $\begin{array}{l}\text { Original } \\
\text { s'arrêtant }\end{array}$ & $\begin{array}{l}\text { AS. } \\
\text { se detuvo }\end{array}$ & $\begin{array}{l}\text { pretérito / } \\
\text { p. simple }\end{array}$ & $\begin{array}{l}\text { TU. } \\
\text { se detuvo }\end{array}$ \\
\hline s'interrompant & $\begin{array}{l}\text { se } \\
\text { interrumpió }\end{array}$ & $»$ & se interrumpió \\
\hline restant & se quedó & $»$ & se quedó \\
\hline regardant & mirando & gerundio & mirando \\
\hline frappant & golpeando & $»$ & golpeando \\
\hline disant & diciendo & $»$ & diciendo \\
\hline n'achevant & terminaba & imperfecto & terminando \\
\hline $\begin{array}{l}\text { continuant } \\
\text { à frapper }\end{array}$ & $\begin{array}{l}\text { seguía } \\
\text { golpeando }\end{array}$ & $»$ & $\begin{array}{l}\text { siguiendo } \\
\text { golpeando }\end{array}$ \\
\hline n'attendant & sin esperar & infinitivo & sin esperar \\
\hline cessant & dejando & gerundio & dejando \\
\hline
\end{tabular}

Était-il possible d'utiliser, dans tous les cas, l'équivalent espagnol du participe présent? Oui, à ceci près que son usage aurait été beaucoup moins naturel. En espagnol, celui-ci (le gerundio) est moins fréquent qu'en français.

De plus, les solutions adoptées par les traducteurs ont des inconvénients notables puisqu'ils changent les valeurs aspectuelles (formes ponctuelles pour traduire des valeurs duratives); redoublement sémantique, seguía golpeando, et plus encore: siguiendo golpeando; la construction infinitive sin esperar pour n'attendant. C'est peut-être encore, hormis le gerundio, l'utilisation de l'imparfait qui s'approche le plus de la valeur du participe présent. 